\title{
Seasonal changes in abundance, lipid and fatty acid composition of Calanus euxinus in the South-eastern Black Sea
}

\author{
N. SEN OZDEMIR ${ }^{1 *}$, A. M. FEYZIOGLU², F. CAF³ AND I. YILDIZ ${ }^{4}$ \\ ${ }^{1}$ Department of Fisheries, Faculty of Agriculture, Bingol University, 120 00, Bingol, Turkey \\ ${ }^{2}$ Department of Marine Sciences and Technology Engineering, Surmene Faculty of Marine Sciences \\ Karadeniz Technical University, 615 30, Trabzon, Turkey \\ ${ }^{3}$ Department of Veterinary Medicine, Vocational School of Technical Sciences, Bingol University, 120 00, Turkey \\ ${ }^{4}$ Marine Sciences and Technology Institute, Karadeniz Technical University,Trabzon, 610 00, Turkey \\ e-mail:nsozdemir@bingol.edu.tr
}

\begin{abstract}
Seasonal changes in abundance, lipid and fatty acid composition of Calanus euxinus Hulsemann, 1991 were analysed monthly during the period from March 2012 to February 2013. The highest abundance of $C$. euxinus was recorded in February $\left(847\right.$ ind. $\mathrm{m}^{-3}$ ) during the sampling period. Female and male C. euxinus peaked in February $\left(587\right.$ ind. $\mathrm{m}^{3}$, 169 ind. $\mathrm{m}^{-3}$, respectively). However, copepodites peaked in November (107 ind. $\mathrm{m}^{-3}$ ). Average total lipid content was determined as percentage $(\%)$ and per individual $\left(\mathrm{mg} \mathrm{ind}^{-1}\right)$. It was proportionally highest in February $(7.03 \%)$ and lowest in September (3.02\%). However, average lipid content per individual was highest in February $\left(0.11 \mathrm{mg}^{-1} \mathrm{in}^{-1}\right)$ and lowest in September and November $\left(0.04 \mathrm{mg} \mathrm{ind}^{-1}\right)$. Major fatty acids in C. euxinus were identified as 16:0, 16:1 n-7, EPA and DHA. $\sum$ SFA, $\sum$ MUFA, $\sum$ PUFA and $\sum$ HUFA were observed to be correlated with temperature. $\sum$ SFA and $\sum$ MUFA increased with the rise in temperature $\left(\mathrm{r}^{2}=0.74, \mathrm{r}^{2}=0.73, \mathrm{p}<0.05\right.$, respectively) whereas $\sum$ PUFA and $\sum$ HUFA increased as temperature decreased $\left(\mathrm{r}^{2}=-0.73, \mathrm{r}^{2}=-0.80\right.$, respectively, $\left.\mathrm{p}<0.05\right)$. Additionally, while $\sum$ PUFA and $\sum$ HUFA increased $\left(\mathrm{r}^{2}=0.61, \mathrm{r}^{2}=-0.68\right.$, respectively, $\mathrm{p}<0.05), \sum$ MUFA decreased $\left(\mathrm{r}^{2}=-0.68, \mathrm{p}<0.05\right)$ as chlorophyll-a increased. It was observed that the degree of unsaturation increased as temperature decreased. Results of the study revealed that C. euxinus has rich lipid content as well as fatty acid composition and it plays an important role in the South-eastern Black Sea ecosystem functionalities especially having key role in energy fluxes to higher trophic levels.
\end{abstract}

Keywords: Abundance, Black Sea, Calanus euxinus, Chlorophyll-a, HUFA, PUFA, Total lipids

\section{Introduction}

Fatty acids (FA) are among the most important molecules transferred from plants to animals in the aquatic food web. Certain classes of FA, such as the omega-3 (n-3) highly unsaturated fatty acids (HUFA) are available in limited quantities and very important for herbivorous zooplankton (Muler-Navarra 1995a; Muler-Navarra et al., 2000; Ravet et al., 2003). These molecules are also of critical importance for the growth and resistance to disease in juvenile and larval fish (Adams, 1999; Olsen, 1999; Sargent et al., 1999). Docosahexaenoic acid (DHA, 22:6 n-3), eicosapentaenoic acid (EPA, 20:5 n-3) and arachidonic acid (ARA, 20:4 n-6) are essential fatty acids (EFA) for many marine species. Due to competitive interactions between DHA and EPA as well as between EPA and ARA, the importance of considering the relative amounts of DHA, EPA and ARA has been demonstrated simultaneously (McEvoy et al., 1998; Estevez et al., 1999; Sargent et al., 1999).
Information about FA may also help with the interpretation of trophic relationships in aquatic systems (Dalsgard et al., 2003) that occur due to the variations in specific fatty acid compositions of primary producers (Volkman et al., 1989; Ahlgren et al., 1992). Thus, it is important to know the FA composition of the zooplankton, which is determined by taxonomic affiliation, changed by diet and modified by starvation or temperature (Arts et al., 2009). Additionally, the need for accumulation of knowledge about the nutritional importance of FA helps us determine the food quality of the fish species with economic significance since the nutritional impact of FA are conveyed from fish to the human through the food web (Simopoulos, 1999; Arts et al., 2001).

Most of the research along the northern regions of the world's oceans focus on copepods (Marshall and Orr, 1972; Mauchline, 1998; Bonnet et al., 2005). Calanus euxinus Hulsemann, 1991 is one of the most important copepod species involved in the pelagic food chain of the Black Sea (Vinogradov et al., 1992). It is a dominant 
secondary producer in the Black Sea, accounting for over one-third of the total zooplankton biomass (Vinogradov et al., 1992). It has an important role in transferring the organic matter from primary producers to the higher taxa. It is estimated that 14.5 and $9.5 \%$ of primary production was consumed by female $C$. euxinus in April and September 1995 in South-western Black Sea, respectively (Besiktepe et al., 1998). Another important feature of C. euxinus is that a part of the population always remained in the deepest part of the oxygenated zone and formed the majority of the permanent zooplankton biomass just in the suboxic zone.

C. euxinus is also the main food for a variety of commercially important fish species, e.g., sprat and anchovy in the Black Sea (Sirotenko and Danilevsky, 1977; Sirotenko and Sorokalit, 1979; Avsar, 1993). It is also a link between the phytoplankton and many fish species, especially including commercially important fish species (Vinogradov et al., 1992, Kovalev et al., 1998). One of the most important commercial fishes in the Black Sea region is anchovy $(85.64 \%$ of the total catch) (TUIK, 2008). Anchovy mainly feed on a variety of phytoplankton and copepod species (Calanus euxinus, Paracalanus parvus) (Bat and Satilmis, 2010). Additionally, Calanus development strongly depends upon seasonal changes in phytoplankton concentration (Ceballos et al., 2004; Rey-Rassat et al., 2004). Therefore, we used chlorophyll-a (chl-a) concentration as the main criteria of the phytoplankton biomass. The present study attempted to reveal the factors that have an effect on the seasonal variation in fatty acid composition and population structure of $C$. euxinus as well as to study the effects on the change of quality and quantity of the fatty acid composition with respect to chl-a, which is an indicator of phytoplankton biomass.

\section{Materials and methods}

\section{Sampling}

The study was performed in the southern part of the Black Sea at a coastal station with coordinates $40^{\circ} 57^{\prime} 12^{\prime \prime} \mathrm{N}$, $-40^{\circ} 9^{\prime} 30^{\prime \prime} \mathrm{E}$ (Fig. 1). Each of the individual samplings was made on a single day for every corresponding month between March 2012 and February 2013 aboard KTU's research vessel Yakamoz. The water depth was $250 \mathrm{~m}$. Water samples (2 l) were collected using Nansen bottles on the surface layer. Temperature and salinity were measured with a conductivity-temperature-depth-oxygen CTD profiler (CTD, General Oceanic Idronaut 316). Zooplankton samples were collected with a vertical haul from the depth of the upper border of the anoxic layer with $\sigma \mathrm{t}=16.2(130 \mathrm{~m})$ up to the surface layer with a $200 \mu \mathrm{m}$ mesh Hydro-Bios net with a mouth diameter of $110 \mathrm{~cm}$.

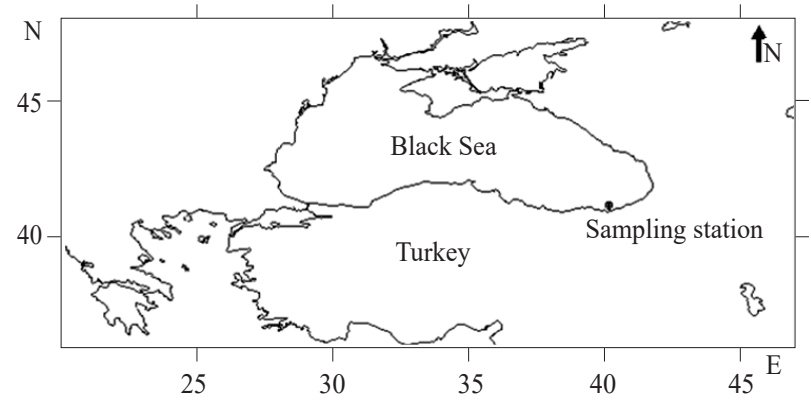

Fig. 1. Sampling station in Camburnu Bay, Trabzon

To determine lipid amount and fatty acid content, samples of copepodites and adults were collected, transferred in to glass vials with screw caps and then stored in $-80^{\circ} \mathrm{C}$ freezer (NUAIRE, USA) until analysis.

\section{Chlorophyll-a (chl-a) analysis}

Seawater samples (2 1) were collected from the surface layer for chl-a assay. Collected samples were filtered through a $0.45 \mu \mathrm{m}$ cellulose acetate membrane and a few drops of magnesium carbonate solution was added to prevent the acidisation on the filter paper. After the filtering process was completed, the filter papers were folded and placed in $15 \mathrm{ml}$ centrifuge tubes. Then the samples were immediately frozen at $-20^{\circ} \mathrm{C}$ and kept until analysis. For analysis, $10 \mathrm{ml} \mathrm{90 \%} \mathrm{acetone}$ solution was added and kept in refrgerator at $4{ }^{\circ} \mathrm{C}$ for $24 \mathrm{~h}$ to ensure transition of chl to acetone. The solution was centrifuged for about $20 \mathrm{~min}$ at $5000 \mathrm{rpm}$ and the supernatant solution was considered for determination of chlorophyll pigment. The absorbance values of the samples at 750, 664, 647, $630 \mathrm{~nm}$ wavelengths were determined using spectrophotometer (SHIMADZU UV 2100). All the extinction values were corrected for a small turbidity blank by subtracting the $750 \mathrm{~nm}$ signal from all the optical densities. Finally, the phytoplankton pigments were estimated as per the following expression of Parsons et al, (1984):

$$
\mathrm{Chl} \mathrm{a}=11.85 \mathrm{OD}_{664}-1.54 \mathrm{OD}_{647}-0.08 \mathrm{OD}_{630}
$$

Values obtained from the equations were then multiplied by volume of the extract $(\mathrm{ml})$ and divided by volume of water (L) filtered, to express chlorophyll content in $\mu \mathrm{g} \mathrm{l}^{-1}$.

\section{Population structure of Calanus euxinus}

C. euxinus individuals were counted under Olympus $\mathrm{BH}_{2}$ stereo microscope (USA) during the sampling period. Abundance was calculated as the number of individuals per $\mathrm{m}^{3}$ (ind. $\mathrm{m}^{-3}$ ). Quantitative analyses of C. euxinus were performed using $3 \mathrm{ml}$ subsamples. Counts were repeated on 4 subsamples (Harris et al.,, 2000). Adult copepods 
(females and males), copepodites and copepod nauplii were identified to species or genus level (Mauchline et al., 1998; Johnson and Allen, 2005).

\section{Total lipid and fatty acid analysis}

Lipids were quantitatively extracted from the samples using chloroform/methanol with a mixing ratio of 2:1 (Folch et al., 1957). About 0.5-1.3 g wet zooplankton samples (WW) was used for lipid extraction per replicates. Weight of copepods used was 0.09-1.6 mg ind ${ }^{-1}$ and numbers of copepods ranged between 2600-3500 individuals during the sampling period. Samples were thoroughly mixed on a magnetic stirrer (Wisd WiseStir MS-MP4) for $2 \mathrm{~h}$ at room temperature. The homogenate was filtered and the solvent was washed with a solution containing $20 \% 0.9 \mathrm{~N} \mathrm{NaCl}$ and stored overnight for further separation in the freezer. On the following day, the separation is expected to yield two phases: the upper phase and the lower phase containing the chloroform and lipids of interest. After discarding the upper phase, chloroform in the lower phase was evaporated under vacuum in a rotary evaporator (IKA-RV 06 ML-US) thereby restoring the lipids. After evaporation of chloroform, the remaining dry lipid was weighed and dry lipid weight (DW) was determined. Total lipids $\left(\%\right.$ and $\left.\mathrm{mg}_{\text {ind }}{ }^{-1}\right)$ were calculated per copepod using the equations:

Total lipid $(\%)=\frac{\text { Wet weight }(\mathrm{WW})}{\text { Dry lipid weight }(\mathrm{DW})} \times 100$

Wet weight: Wet zooplankton sample weight (g)

Dry weight: After evaporation of chloroform, the remaining dry lipid weight $(\mathrm{g})$

Average individual weight $(\mathrm{mg})=\frac{\text { Total sample weight }(\mathrm{mg})}{\text { No. of individual in total samples }}$

Total lipid $\left(\mathrm{mg}\right.$ ind $\left.^{-1}\right)=$ Average individual weight $(\mathrm{mg}) \times$ Total lipid (\%)

To determine FAME (fatty acid methyl esters) of total lipids, $2 \mathrm{ml}$ chloroform and $1 \mathrm{ml} 0.21 \mathrm{~N} \mathrm{NaOH}$ in methanol was added in the extracted lipid sample and the solution was mixed for about $2 \mathrm{~h}$ on a magnetic stirrer. Then, 0.5-0.7 $\mathrm{ml}$ of $0.5 \mathrm{~N} \mathrm{CH}_{3} \mathrm{COOH}$ in distilled water was added and the mixture was stored in a freezer. Following the removal of upper phase, the liquid portion of lower phase (chloroform) was evaporated, $2 \mathrm{ml}$ hexane was added on the restored dry lipid and the sample was transferred to a vial (Kates, 1986). The FAME were detected by Shimadzu GC-17 ver 3 Gas Chromatograph (GC) (Kyoto, Japan). For this analysis, capillary columns with a length of $25 \mathrm{~m}$, an inner diameter of $0.25 \mu \mathrm{m}$ and a thickness of $25 \mu \mathrm{m}$ (Permabond) were used (MachereyNagel, Germany). Twenty microlitre sample was used to inject into the GC. During analysis, the column temperature was set to $120-220^{\circ} \mathrm{C}$ using increments of $5^{\circ} \mathrm{C}$ per min until $200^{\circ} \mathrm{C}$ was reached and $4^{\circ} \mathrm{C}$ per min to $220^{\circ} \mathrm{C}$. The column was kept for $8 \mathrm{~min}$ at $220^{\circ} \mathrm{C}$ and the total time was determined to be $35 \mathrm{~min}$. The injection temperature was set to $240^{\circ} \mathrm{C}$ and detector temperature to $280^{\circ} \mathrm{C}$. Nitrogen was used as the carrier gas (Christie, 1990).

\section{Statistical analysis}

Data were analysed using STATISTICA 8.0. One-way analysis of variance (ANOVA) was used to test significant differences between the samples. The ANOVA critical significance value " $p$ ", was given in the text to indicate the level of difference (Stoline, 1981).

\section{Results and discussion}

\section{Hydrography}

Vertical profiles of the water salinity $\left(\mathrm{g} \mathrm{l}^{-1}\right)$ and temperature obtained during the sampling are shown in Fig. 2 and 3. Over the sampling period, sea surface temperatures fluctuated from $8.84^{\circ} \mathrm{C}$ in March to $27.75^{\circ} \mathrm{C}$ in August and a seasonal thermocline was observed to start at a depth of $20 \mathrm{~m}$ in April. Water temperature at a depth of $40 \mathrm{~m}$ decreased to $6.8^{\circ} \mathrm{C}$ and the cold intermediate layer (CIL) was observed. CIL is a well-known feature of the Black Sea thermohaline structure (Oguz et al., 1992, Oguz et al., 1999). CIL, marked by temperatures

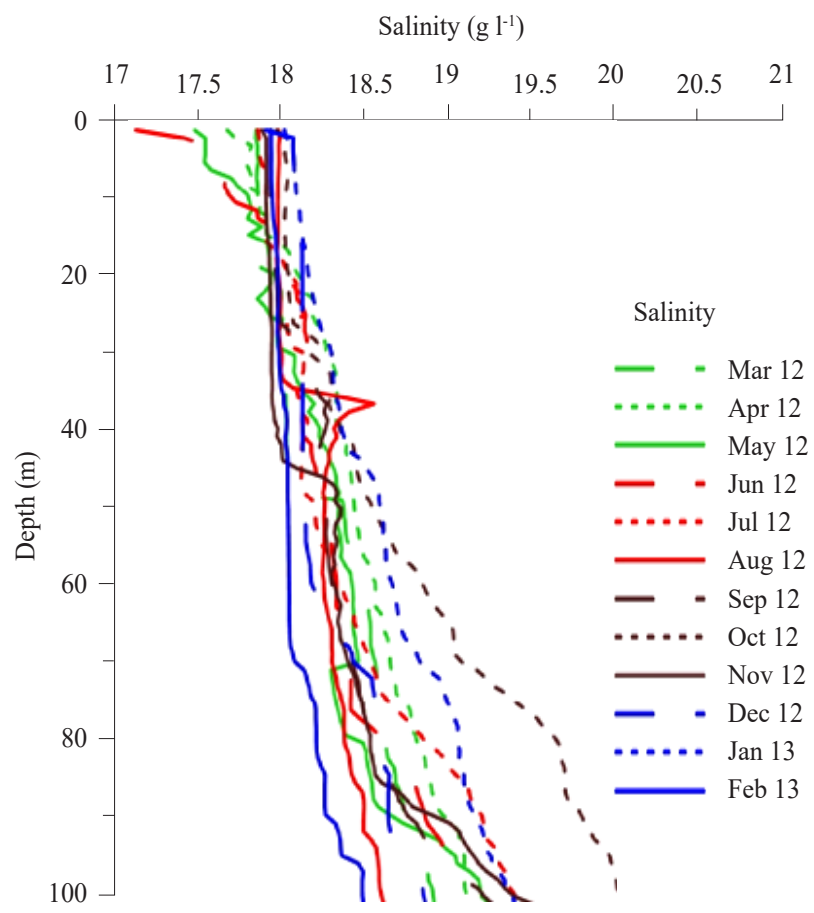

Fig. 2. Vertical distribution of salinity $\left(\mathrm{g} \mathrm{l}^{-1}\right)$ at sampling station during the sampling period 


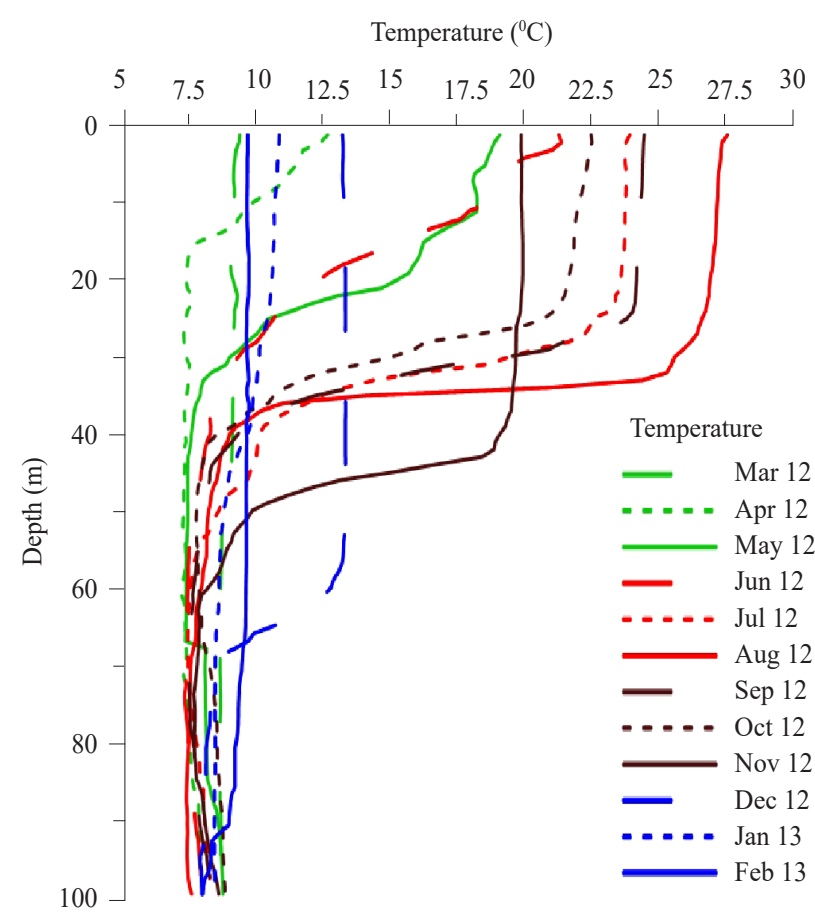

Fig. 3. Vertical distribution of temperature $\left({ }^{\circ} \mathrm{C}\right)$ at sampling station during the sampling period

lower than $8^{\circ} \mathrm{C}$, is a unique water mass of the upper layer thermohaline structure. It is formed by the convective processes associated with the winter cooling of surface waters (Tomazin, 1985; Ovchinnikov and Popov, 1987). In the surface layer, the lowest salinity was observed at $17.14 \mathrm{~g} \mathrm{l}^{-1}$ in June, indicating that runoff and/or rainfall influenced water salinity, whereas the highest salinity was measured at $17.96 \mathrm{~g} \mathrm{l}^{-1}$ in August. Additionally, salinity increased depending on the depth and at $100 \mathrm{~m}$ it ranged from $18.70 \mathrm{~g} \mathrm{l}^{-1}$ in August to $20.1 \mathrm{~g} \mathrm{l}^{-1}$ in October. The CTD profiles (salinity, temperature) from March and April are significantly different from those of the rest of the year. Bottom water of the Black Sea is more saline than the surface water since the denser water of the Mediterranean reaches the Black Sea through the straits (Latif et al., 1991).

\section{Chl-a concentration}

The mean integrated concentration of chl-a during sampling season was $1.08 \mu \mathrm{g} \mathrm{l}^{-1}$. Chl-a was lowest in April $\left(0.54 \mu \mathrm{g} \mathrm{l}^{-1}\right)$ and the highest in December $\left(1.82 \mu \mathrm{g} \mathrm{l}^{-1}\right)$. While chl-a was $1.41 \mu \mathrm{g} \mathrm{l}^{-1}$ in March, it decreased sharply in April and increased again to $1.65 \mathrm{\mu g}^{-1}$ in May. In June, the amount of chl-a was $1.16 \mu \mathrm{g} \mathrm{l}^{-1}$ and it exhibited a homogeneous structure from July upto November. This suggested that spring peak occurs later in the spring (May; $1.65 \mu \mathrm{g} \mathrm{l}^{-1}$ ) whereas autumn peak is in November (Fig. 4).

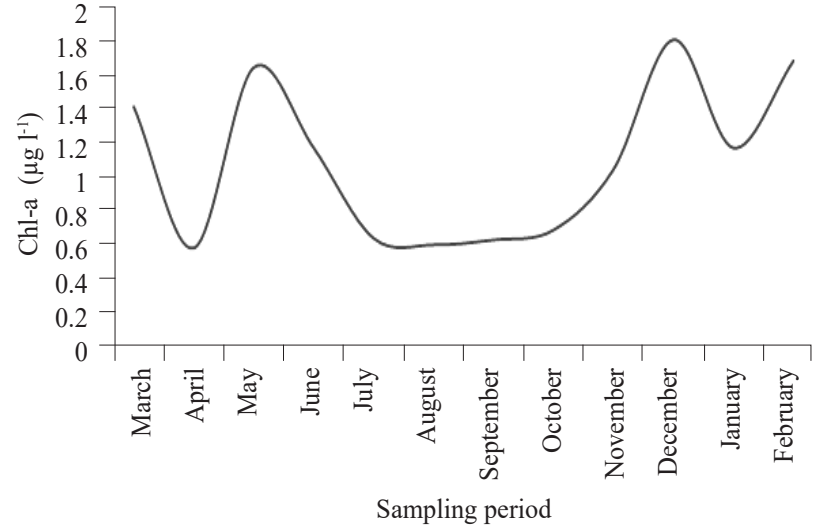

Fig. 4. Chl-a concentration during sampling period

Population structure of C. euxinus

The highest abundance for $C$. euxinus was recorded in February (847 ind $\mathrm{m}^{-3}$ ) and the least abundance in March (37 ind. $\mathrm{m}^{-3}$ ). Seasonally $C$. euxinus reached the most average total abundance in winter (1063 ind. $\mathrm{m}^{-3}$ ) and the least abundance was in spring (256 ind. $\mathrm{m}^{-3}$ ) (Fig. 5). Female C. euxinus peaked in February (587 ind. $\mathrm{m}^{-3}$ )during the sampling period. It was $70 \%$ of the total abundance in February. Female $C$. euxinus was the least abundant in June (13 ind. $\mathrm{m}^{-3}$ ). Similarly, male C. euxinus peaked in February (169 ind. $\mathrm{m}^{-3}$ ) and it was $20 \%$ of the total abundance in February. Male C. euxinus was least abundant with same value in March and August (5 ind. $\mathrm{m}^{-3}$ ). However, copepodites reached the highest abundance in November (107 ind $\mathrm{m}^{-3}$ ) and were the least abundant in March (14 ind. $\mathrm{m}^{-3}$ ) (Fig. 6).

\section{Total lipids}

Table 1 represents the seasonal variations in total lipids of Calanus euxinus (wet weight, WW) in terms of per individual $\left(\mathrm{mg}\right.$ ind $\left.^{-1}\right)$ and percentage (\%) during

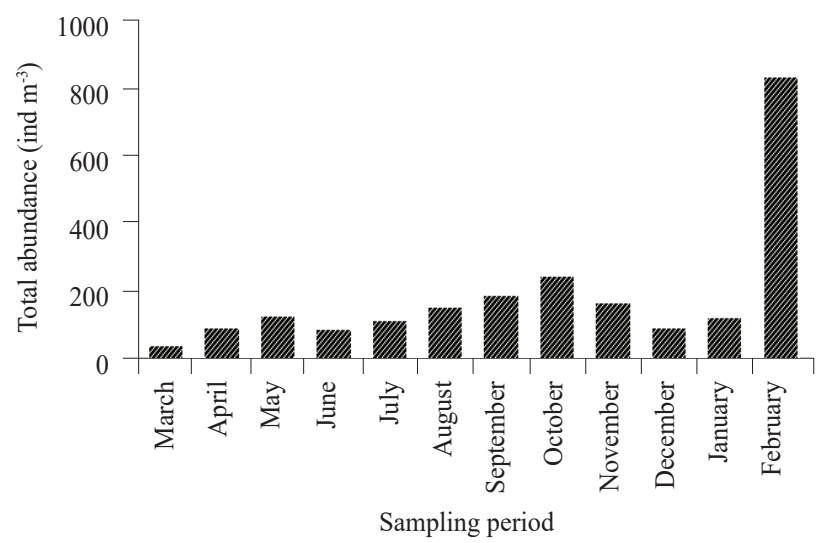

Fig. 5. Total abundance of C. euxinus during the sampling period 


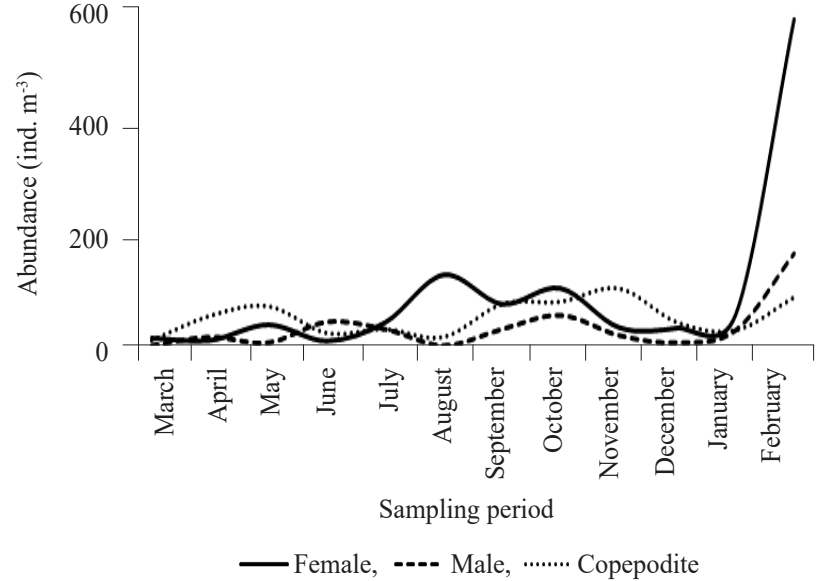

Fig. 6. Abundance of Calanus euxinus male, female and copepodites during the sampling period behenic acid (22:0). The highest values of behenic acid and myristic acid were measured in November (7.61 and $13.30 \%$ respectively) while palmitic acid was highest in April (12.34\%). Additionally, the lowest values of behenic acid and myristic acid were observed in April ( $2.84 \%$ and $3.34 \%$ respectively) while palmitic acid was lowest in July (9.48\%). Total monounsaturated fatty acids ( $\sum$ MUFA) ranged from 16.39-29.95\% during the sampling period, with the highest $\sum$ MUFA in September and the lowest in March. The main components of $\sum$ MUFA were palmitoleic acid (16:1 n-7) and oleic acid (18:1 n-9c). Palmitoleic acid levels ranged from 3.34 to $9.85 \%$. The highest values of palmitoleic acid and oleic acid were recorded in September (9.85\% and 5.93\%, respectively) while the lowest figure was observed in March (3.34\% and $3.07 \%$, respectively). The total polyunsaturated fatty acids

Table 1. Total lipid $\left(\%\right.$ and $m g$ ind $\left.^{-1}\right)$ content of Calanus euxinus during the sampling period

\begin{tabular}{lllllll}
\hline Total lipid & March & April & May & June & July & August \\
\hline$\%$ & $5.61 \pm 0.31^{\mathrm{abc}}$ & $4.43 \pm 0.35^{\mathrm{abc}}$ & $5.50 \pm 1.68^{\mathrm{abc}}$ & $4.37 \pm 1.76^{\mathrm{abc}}$ & $6.84 \pm 2.90^{\mathrm{a}}$ & $6.26 \pm 1.27^{\mathrm{ac}}$ \\
$\mathrm{mg} \mathrm{ind}^{-1}$ & $0.09 \pm 0.01^{\mathrm{d}}$ & $0.06 \pm 0.01^{\mathrm{b}}$ & $0.05 \pm 0.02^{\mathrm{ab}}$ & $0.07 \pm 0.03^{\mathrm{c}}$ & $0.09 \pm 0.04^{\mathrm{d}}$ & $0.09 \pm 0.02^{\mathrm{d}}$ \\
\hline Total lipid & September & October & November & December & January & February \\
\hline$\%$ & $3.02 \pm 0.36^{\mathrm{b}}$ & $5.72 \pm 0.42^{\mathrm{abc}}$ & $3.24 \pm 0.22^{\mathrm{bc}}$ & $4.80 \pm 1.31^{\mathrm{abc}}$ & $5.10 \pm 0.13^{\mathrm{abc}}$ & $7.03 \pm 2.04^{\mathrm{a}}$ \\
$\mathrm{mg} \mathrm{ind}^{-1}$ & $0.04 \pm 0.01^{\mathrm{a}}$ & $0.09 \pm 0.01^{\mathrm{d}}$ & $0.04 \pm 0.01^{\mathrm{d}}$ & $0.07 \pm 0.02^{\mathrm{c}}$ & $0.08 \pm 0.01^{\text {cd }}$ & $0.11 \pm 0.03^{\mathrm{e}}$ \\
\hline
\end{tabular}

$(\mathrm{n}=3)$, values are means \pm standard deviation

Means followed by different letters and letter groups in the same row are significantly different $(\mathrm{p}<0.05)$

the sampling period which spans from March 2012 to February 2013. The percentage of total lipids changed depending on the sampling period. Total lipids by percentage and total lipids by $\mathrm{mg}$ ind $^{-1}$ showed a significant linear relationship (total lipid by $\mathrm{mg}$ ind $^{-1}=0.02$, total lipid by $\left.\%=0.01, \mathrm{r}^{2}=0.78, \mathrm{p}<0.05\right)$. The average levels of total lipids by percentage during March, April, May, June, October, December and January were found to be close to each other whereas the levels in July and September differed from the other months $(\mathrm{p}<0.05)$. Moreover, total lipids (both by $\%$ and $\mathrm{mg}$ ind $^{-1}$ ) reached the highest level in February $\left(7.03 \%, 0.11 \mathrm{mg}^{\text {ind }^{-1}}\right)$, total lipids by percentage was at its lowest level in September (at 3.02\%) and the total lipids by $\mathrm{mg}$ ind $^{-1}$ were lowest in September and November $\left(0.04 \mathrm{mg}\right.$ ind $\left.^{-1}\right)$.

\section{Fatty acid profile}

Table 2 represents the changes in FA profile (percent of total FA) of Calanus euxinus during the sampling period (March 2012-February 2013). The total saturated fatty acids ( $\sum$ SFA) contributed $23.47-37.71 \%$ of the total FA, the lowest value of which was obtained in March and the highest in November. The main component was palmitic acid (16:0) followed by myristic acid (14:0) and
( $\sum$ PUFA) fluctuated within a wide range. The highest value for $\sum$ PUFA was detected in March at $60.14 \%$, while the lowest level was measured in November at $33.91 \%$. EPA and DHA were the two PUFA detected mainly regardless of the sampling period. Other fatty acids that are important in aquatic ecosystems are highly unsaturated fatty acids (HUFA). The highest value of $\sum$ HUFA was recorded in March $(57.73 \%)$ while palmitic acid (16:0) was highest in April (12.34\%) and lowest in July $(9.48 \%)$.

Marine zooplankton are a very diverse group in the world's oceans with numerous taxa of high abundance and biomass. Many of these zooplankton species, in particular the dominating copepods, are able to accumulate large reserves of energy-rich lipids (Lee et al., 1971b; Lee and Hirota, 1973). These accumulated amounts of lipids are the major pathways for marine food webs (Dalsgaard et al., 2003). C. euxinus is a dominant component of the zooplankton biomass in the Rim Current system of the Black Sea (Svetlichny et al., 2009). It has adapted to the low salinity of the Black Sea waters (18 to 20\%o). Preadults and adults undergo diel vertical migration within the aerobic layer (0 to $150 \mathrm{~m}$ ) 
Table 2. Fatty acid composition in Calanus euxinus during the sampling period ( $\%$ total defined FAME)

\begin{tabular}{|c|c|c|c|c|c|c|}
\hline Fatty acids & March & April & May & June & July & August \\
\hline $10: 0$ & $0.71 \pm 0.06^{\mathrm{abc}}$ & $1.04 \pm 0.01^{\mathrm{b}}$ & - & $0.86 \pm 0.31^{\mathrm{ab}}$ & $0.71 \pm 0.08^{\mathrm{abc}}$ & $0.79 \pm 0.02^{\mathrm{abc}}$ \\
\hline $12: 0$ & - & - & - & - & - & - \\
\hline $14: 0$ & $3.34 \pm 0.02^{\mathrm{f}}$ & $7.47 \pm 1.10^{\mathrm{a}}$ & $10.34 \pm 0.16^{\mathrm{ce}}$ & $10.80 \pm 0.84^{\mathrm{cd}}$ & $12.85 \pm 0.26^{\mathrm{b}}$ & $10.67 \pm 0.51^{\mathrm{cd}}$ \\
\hline $15: 0$ & $0.61 \pm 0.13^{\mathrm{f}}$ & - & $1.40 \pm 0.10^{\text {ce }}$ & $1.11 \pm 0.09^{\text {ad }}$ & $1.39 \pm 0.07^{\text {bce }}$ & $1.23 \pm 0.10^{\mathrm{abc}}$ \\
\hline $16: 0$ & $12.00 \pm 0.37^{\mathrm{ab}}$ & $12.34 \pm 0.01^{\mathrm{b}}$ & $11.04 \pm 0.13^{\mathrm{abcd}}$ & $10.61 \pm 0.80^{\text {acd }}$ & $9.48 \pm 0.30^{\mathrm{d}}$ & $11.60 \pm 0.94^{\mathrm{abc}}$ \\
\hline $17: 0$ & $1.26 \pm 0.21^{\mathrm{d}}$ & - & $0.77 \pm 0.08^{\mathrm{bc}}$ & $0.37 \pm 0.06^{\mathrm{a}}$ & $0.99 \pm 0.03^{\mathrm{c}}$ & $0.60 \pm 0.05^{\mathrm{ab}}$ \\
\hline $18: 0$ & $2.71 \pm 0.33^{\mathrm{f}}$ & $2.52 \pm 0.05^{\text {ef }}$ & $1.63 \pm 0.01^{\mathrm{abcd}}$ & $1.89 \pm 0.19^{\text {cde }}$ & $1.28 \pm 0.12^{\mathrm{abcd}}$ & $1.10 \pm 0.23^{\mathrm{ab}}$ \\
\hline $20: 0$ & - & - & - & - & - & $1.35 \pm 0.28^{\mathrm{ab}}$ \\
\hline 21:0 & - & - & - & - & - & - \\
\hline $22: 0$ & $2.84 \pm 0.10^{\mathrm{f}}$ & $4.63 \pm 0.26^{\mathrm{de}}$ & $5.22 \pm 0.24^{\text {abe }}$ & $7.09 \pm 0 ., 61^{\mathrm{c}}$ & $7.50 \pm 0.13^{\mathrm{c}}$ & $4.15 \pm 0.02^{\mathrm{d}}$ \\
\hline $24: 0$ & - & - & - & - & - & - \\
\hline$\sum \mathrm{SFA}$ & $23.47 \pm 0.41^{g}$ & $28.00 \pm 0.90^{\mathrm{d}}$ & $30.40 \pm 0.43^{\mathrm{abd}}$ & $32.73 \pm 1.04^{\text {ace }}$ & $34.20 \pm 0.43^{\text {ce }}$ & $31.49 \pm 0.38^{\mathrm{abc}}$ \\
\hline $14: 1$ & - & - & - & - & - & - \\
\hline $15: 1$ & - & $1.25 \pm 0.01^{\mathrm{cd}}$ & $1.06 \pm 0.08^{\mathrm{abc}}$ & $1.03 \pm 0.04^{\mathrm{d}}$ & $1.34 \pm 0.08^{\mathrm{ab}}$ & $0.91 \pm 0.07^{\mathrm{abc}}$ \\
\hline $16: 1 \mathrm{n}-7$ & $3.34 \pm 0.37^{\mathrm{b}}$ & $9.79 \pm 0.02^{\mathrm{a}}$ & $5.11 \pm 0.31^{\text {cde }}$ & $5.59 \pm 0.69^{\mathrm{de}}$ & $7.52 \pm 0.40^{\mathrm{f}}$ & $9.34 \pm 0.77^{\mathrm{ag}}$ \\
\hline $17: 1$ & $0.57 \pm 0.09^{\mathrm{a}}$ & $1.26 \pm 0.03^{b}$ & $0.86 \pm 0.05^{\mathrm{ab}}$ & $0.90 \pm 0.02^{\mathrm{ab}}$ & $0.99 \pm 0.03^{\mathrm{ab}}$ & $0.89 \pm 0.15^{\mathrm{ab}}$ \\
\hline $18: 1 \mathrm{n}-9 t$ & - & - & - & - & - & $1.01 \pm 0.02^{\mathrm{ab}}$ \\
\hline $18: 1 \mathrm{n}-9 \mathrm{c}$ & $3.07 \pm 0.29^{c}$ & $4.26 \pm 0.24^{\mathrm{ad}}$ & $4.23 \pm 0.03^{\mathrm{ad}}$ & $5.15 \pm 0.08^{\mathrm{a}}$ & $5.51 \pm 0.05^{\text {be }}$ & $4.81 \pm 0.20^{\mathrm{ef}}$ \\
\hline $18: 1 \mathrm{n}-7$ & $0.68 \pm 0.14^{\mathrm{a}}$ & $0.90 \pm 0.01^{b}$ & $0.59 \pm 0.01^{\mathrm{a}}$ & - & - & - \\
\hline $20: 1$ n-9 & $1.98 \pm 0.02^{b}$ & $2.81 \pm 0.44^{\mathrm{bd}}$ & $2.92 \pm 0.04^{\text {bde }}$ & $4.28 \pm 0.63^{\mathrm{ac}}$ & $4.41 \pm 0.28^{\mathrm{ac}}$ & $4.02 \pm 0.63^{\mathrm{ae}}$ \\
\hline $20: 1 \mathrm{n}-\mathrm{X}$ & $4.64 \pm 0.12^{\mathrm{abcd}}$ & $4.16 \pm 0.12^{\mathrm{acd}}$ & $5.03 \pm 0.07^{\mathrm{abd}}$ & $5.27 \pm 0.63^{\mathrm{ab}}$ & $5.88 \pm 0.06^{\mathrm{b}}$ & $4.17 \pm 0.63^{\mathrm{acd}}$ \\
\hline $22: 1 n-9$ & $1.31 \pm 0.05^{\mathrm{d}}$ & $0.90 \pm 0.01^{\mathrm{ac}}$ & $0.86 \pm 0.06^{\mathrm{abc}}$ & $0.79 \pm 0.01^{\mathrm{abc}}$ & $0.85 \pm 0.02^{\mathrm{abc}}$ & $0.59 \pm 0.01^{\mathrm{bc}}$ \\
\hline $24: 1$ & $0.80 \pm 0.03^{\mathrm{d}}$ & - & $0.49 \pm 0.01^{\mathrm{a}}$ & - & - & - \\
\hline$\sum$ MUFA & $16.39 \pm 0.42^{\mathrm{e}}$ & $25.33 \pm 0.71^{\mathrm{bc}}$ & $21.15 \pm 0.55^{\mathrm{a}}$ & $23.00 \pm 1.80^{\mathrm{ab}}$ & $26.50 \pm 0.81^{\mathrm{abc}}$ & $25.74 \pm 2.29^{\mathrm{bc}}$ \\
\hline $18: 2 \mathrm{n}-6 t$ & - & - & - & $0.48 \pm 0.02^{\mathrm{c}}$ & $0.43 \pm 0.02^{b}$ & - \\
\hline $18: 2 n-6 c$ & $1.78 \pm 0.02^{\mathrm{bc}}$ & $2.02 \pm 0.04^{\mathrm{cd}}$ & $2.43 \pm 0.02^{\mathrm{ad}}$ & $2.38 \pm 0.10^{\text {ad }}$ & $2.66 \pm 0.19^{\mathrm{a}}$ & $2.54 \pm 0.04^{\mathrm{a}}$ \\
\hline $18: 3 n-3 c$ & $0.63 \pm 0.11^{\mathrm{c}}$ & $1.19 \pm 0.02^{\mathrm{a}}$ & $1.46 \pm 0.07^{b}$ & $1.25 \pm 0.07^{\mathrm{a}}$ & $1.48 \pm 0.06^{\mathrm{b}}$ & $1.49 \pm 0.06^{b}$ \\
\hline $18: 3 n-6$ & - & - & $0.34 \pm 0.01^{\mathrm{c}}$ & $0.69 \pm 0.25^{\mathrm{b}}$ & $0.58 \pm 0.11^{\mathrm{bd}}$ & - \\
\hline $20: 2 n-6$ & - & - & $0.45 \pm 0.08^{\mathrm{a}}$ & $0.48 \pm 0.03^{\mathrm{ab}}$ & $0.54 \pm 0.02^{\mathrm{ab}}$ & - \\
\hline $20: 3 n-6$ & - & - & - & - & - & - \\
\hline $20: 4 n-6$ & - & - & - & - & - & - \\
\hline $20: 5$ n-3 (EPA) & $18.05 \pm 0.12^{\mathrm{e}}$ & $16.70 \pm 0.19^{\mathrm{a}}$ & $15.92 \pm 0.09^{\mathrm{a}}$ & $15.66 \pm 0.39^{\mathrm{ac}}$ & $15.72 \pm 0.30^{\mathrm{ac}}$ & $16.41 \pm 0.10^{\mathrm{a}}$ \\
\hline $22: 5 n-3$ & $0.67 \pm 0.04^{\mathrm{a}}$ & - & $0.73 \pm 0.04^{\mathrm{a}}$ & $0.77 \pm 0.04^{\mathrm{a}}$ & $1.01 \pm 0.22^{\mathrm{b}}$ & $0.61 \pm 0.02^{\mathrm{a}}$ \\
\hline 22:6 n-3 (DHA) & $39.01 \pm 0.68^{\mathrm{f}}$ & $26.76 \pm 0.03^{b}$ & $27.12 \pm 0.92^{\mathrm{b}}$ & $22.56 \pm 1.34^{\mathrm{cd}}$ & $16.88 \pm 0.31^{\mathrm{a}}$ & $21.72 \pm 1.99^{c}$ \\
\hline$\sum$ PUFA & $60.14 \pm 0.69^{\mathrm{f}}$ & $46.67 \pm 0.21^{\mathrm{ac}}$ & $48.45 \pm 0.94^{\mathrm{a}}$ & $44.27 \pm 1.48^{\mathrm{ce}}$ & $39.30 \pm 0.44^{b}$ & $42.77 \pm 1.99^{\mathrm{e}}$ \\
\hline$\sum$ HUFA & $57.73 \pm 0.65^{\mathrm{e}}$ & $43.46 \pm 0.70^{\mathrm{a}}$ & $44.22 \pm 1.02^{\mathrm{a}}$ & $39.47 \pm 1.73^{\mathrm{d}}$ & $34.15 \pm 0.10^{\mathrm{b}}$ & $38.74 \pm 2.08^{\mathrm{d}}$ \\
\hline \multirow[t]{2}{*}{ DHA/EPA } & $2.16 \pm 0.05^{\mathrm{f}}$ & $1.60 \pm 0.02^{\mathrm{ab}}$ & $1.70 \pm 0.05^{b}$ & $1.44 \pm 0.05^{\text {aef }}$ & $1.07 \pm 0.02^{\mathrm{c}}$ & $1.32 \pm 0.11^{\mathrm{def}}$ \\
\hline & September & October & November & December & January & February \\
\hline $10: 0$ & $0.40 \pm 0.04^{\text {cd }}$ & $0.52 \pm 0.08^{\mathrm{ac}}$ & $0.60 \pm 0.02^{\mathrm{abc}}$ & $0.86 \pm 0.18^{\mathrm{ab}}$ & $0.81 \pm 0.21^{\mathrm{abc}}$ & $1.98 \pm 0.30^{\mathrm{e}}$ \\
\hline $12: 0$ & - & $0.37 \pm 0.04^{\mathrm{a}}$ & $0.38 \pm 0.03^{\mathrm{a}}$ & - & - & - \\
\hline $14: 0$ & $12.20 \pm 0.11^{\mathrm{bd}}$ & $12.89 \pm 0.34^{\mathrm{b}}$ & $13.30 \pm 0.78^{b}$ & $8.70 \pm 0.56^{\mathrm{a}}$ & $7.73 \pm 1.10^{\mathrm{a}}$ & $7.99 \pm 0.25^{\mathrm{a}}$ \\
\hline $15: 0$ & $1.34 \pm 0.04^{\text {bce }}$ & $1.49 \pm 0.01^{\mathrm{e}}$ & $1.19 \pm 0.01^{\mathrm{abd}}$ & $1.19 \pm 0.05^{\mathrm{abc}}$ & $1.07 \pm 0,09^{\mathrm{ad}}$ & $0.98 \pm 0.02^{\mathrm{d}}$ \\
\hline $16: 0$ & $9.98 \pm 0.32^{\mathrm{cd}}$ & $10.85 \pm 0.11^{\mathrm{abcd}}$ & $10.99 \pm 0.62^{\mathrm{abcd}}$ & $11.64 \pm 0.13^{\mathrm{abcd}}$ & $11.28 \pm 0.41^{\mathrm{abc}}$ & $11.86 \pm 1.22^{\mathrm{ab}}$ \\
\hline $17: 0$ & - & $0.43 \pm 0.06^{\mathrm{a}}$ & $0.39 \pm 0.04^{\mathrm{a}}$ & - & $0.47 \pm 0.05^{\mathrm{a}}$ & $0.77 \pm 0.17^{\mathrm{bc}}$ \\
\hline $18: 0$ & $0.88 \pm 0.08^{\mathrm{a}}$ & $1.22 \pm 0.01^{\mathrm{abc}}$ & $1.24 \pm 0.07^{\mathrm{abc}}$ & $1.83 \pm 0.02^{\text {bcde }}$ & $1.36 \pm 0.20^{\mathrm{abcd}}$ & $2.01 \pm 0.78^{\mathrm{abcd}}$ \\
\hline $20: 0$ & $1.99 \pm 0.02^{\mathrm{d}}$ & - & $1.58 \pm 0.04^{b}$ & $1.26 \pm 0.07^{\mathrm{a}}$ & $0.89 \pm 0.05^{\mathrm{c}}$ & - \\
\hline $21: 0$ & $0.40 \pm 0.02^{\mathrm{a}}$ & - & - & - & - & - \\
\hline $22: 0$ & $5.86 \pm 0.09^{\mathrm{ab}}$ & $7.20 \pm 0.01^{\mathrm{c}}$ & $7.61 \pm 0.30^{c}$ & $4.93 \pm 0.24^{\text {ade }}$ & $6.04 \pm 0.88^{b}$ & $5.91 \pm 0.14^{\mathrm{ab}}$ \\
\hline $24: 0$ & - & $0.22 \pm 0.04^{b}$ & $0.43 \pm 0 .{ }^{2} 2 \mathrm{a}$ & - & - & $0.49 \pm 0.06^{\mathrm{a}}$ \\
\hline$\sum \mathrm{SFA}$ & $33.05 \pm 0.46^{\text {ace }}$ & $35.19 \pm 0.5 \mathrm{1}^{\mathrm{ef}}$ & $37.71 \pm 0.36^{\mathrm{f}}$ & $30.41 \pm 0.76^{\text {abd }}$ & $29.65 \pm 1.31^{\mathrm{bd}}$ & $31.99 \pm 2.54^{\mathrm{abc}}$ \\
\hline
\end{tabular}




\begin{tabular}{|c|c|c|c|c|c|c|}
\hline & September & October & November & December & January & February \\
\hline $14: 1$ & $0.45 \pm 0.03^{b}$ & $0.32 \pm 0.01^{\mathrm{a}}$ & $0.59 \pm 0.01^{\mathrm{c}}$ & - & - & - \\
\hline $15: 1$ & $1.05 \pm 0.05^{\mathrm{abc}}$ & $1.09 \pm 0.04^{\mathrm{bc}}$ & $0.84 \pm 0.02^{\mathrm{a}}$ & - & - & $0.99 \pm 0.23^{\mathrm{ab}}$ \\
\hline $16: 1 \mathrm{n}-7$ & $9.85 \pm 1.05^{\mathrm{a}}$ & $8.04 \pm 0.40^{\mathrm{fg}}$ & $9.42 \pm 0.32^{\mathrm{ag}}$ & $4.66 \pm 0.33^{\mathrm{bcd}}$ & $3.59 \pm 0.11^{\mathrm{bc}}$ & $6.70 \pm 0.83^{\mathrm{ef}}$ \\
\hline $17: 1$ & $1.06 \pm 0.02^{\mathrm{ab}}$ & $1.08 \pm 0.04^{\mathrm{ab}}$ & $1.32 \pm 0.59^{b}$ & $0.57 \pm 0.03^{\mathrm{a}}$ & $0.91 \pm 0.02^{\mathrm{ab}}$ & $1.07 \pm 0.39^{\mathrm{ab}}$ \\
\hline $18: 1 \mathrm{n}-9 t$ & $0.92 \pm 0.06^{\mathrm{a}}$ & - & $0.79 \pm 0.03^{\mathrm{d}}$ & $1.00 \pm 0.07^{\mathrm{ab}}$ & $1.13 \pm 0.04^{c}$ & $1.07 \pm 0.10^{\mathrm{bc}}$ \\
\hline $18: 1 \mathrm{n}-9 \mathrm{c}$ & $5.01 \pm 0.13^{\text {be }}$ & $5.74 \pm 0.21^{\mathrm{f}}$ & $4.67 \pm 0.16^{\mathrm{abd}}$ & $4.12 \pm 0.07^{\mathrm{a}}$ & $3.22 \pm 0.02^{\mathrm{c}}$ & $3.09 \pm 0.30^{c}$ \\
\hline $18: 1 \mathrm{n}-7$ & - & - & - & - & - & - \\
\hline $20: 1$ n-9 & $5.38 \pm 0.17^{\mathrm{cf}}$ & $4.04 \pm 0.13^{\mathrm{a}}$ & $5.67 \pm 0.05^{\mathrm{f}}$ & $3.61 \pm 0.45^{\text {ade }}$ & $4.49 \pm 0.31^{\mathrm{ac}}$ & $2.43 \pm 0.54^{\mathrm{b}}$ \\
\hline $20: 1 \mathrm{n}-\mathrm{X}$ & $5.12 \pm 0.03^{\mathrm{ab}}$ & $4.42 \pm 0.02^{\text {acd }}$ & $3.41 \pm 0.08^{c}$ & $5.25 \pm 0.27^{\mathrm{ab}}$ & $5.88 \pm 0.75^{b}$ & $3.73 \pm 1.08^{\mathrm{cd}}$ \\
\hline $22: 1 n-9$ & $0.58 \pm 0.08^{\mathrm{b}}$ & $0.86 \pm 0.01^{\mathrm{abc}}$ & $0.89 \pm 0.02^{\mathrm{abc}}$ & $0.96 \pm 0.28^{\mathrm{a}}$ & $0.85 \pm 0.22^{\mathrm{abc}}$ & $1.05 \pm 0.06^{\mathrm{ad}}$ \\
\hline $24: 1$ & $0.53 \pm 0.01^{\mathrm{ac}}$ & $0.58 \pm 0.01^{\mathrm{ac}}$ & $0.78 \pm 0.02^{\mathrm{d}}$ & $0.61 \pm 0.06^{\mathrm{c}}$ & $0.51 \pm 0.06^{\mathrm{a}}$ & $0.48 \pm 0.06^{\mathrm{a}}$ \\
\hline$\sum$ MUFA & $29.95 \pm 1.05^{\mathrm{d}}$ & $26.27 \pm 0.12^{\mathrm{abc}}$ & $28.38 \pm 0.36^{\mathrm{cd}}$ & $20.78 \pm 0.90^{\mathrm{a}}$ & $20.58 \pm 1.01^{\mathrm{a}}$ & $20.61 \pm 2.91^{\mathrm{a}}$ \\
\hline $18: 2 \mathrm{n}-6 t$ & - & $0.20 \pm 0.02^{\mathrm{a}}$ & - & - & - & $0.97 \pm 0.42^{\mathrm{b}}$ \\
\hline $18: 2 n-6 c$ & $2.64 \pm 0.08^{\mathrm{a}}$ & $2.49 \pm 0.02^{\mathrm{a}}$ & $1.93 \pm 0.03^{\mathrm{bc}}$ & $1.53 \pm 0.08^{b}$ & $1.57 \pm 0.07^{\mathrm{b}}$ & $1.55 \pm 0.04^{\mathrm{e}}$ \\
\hline $18: 3 n-3 c$ & $1.62 \pm 0.02^{\mathrm{b}}$ & $1.19 \pm 0.01^{\mathrm{a}}$ & $1.16 \pm 0.05^{\mathrm{a}}$ & $1.15 \pm 0.05^{\mathrm{a}}$ & $1.26 \pm 0.11^{\mathrm{a}}$ & $0.74 \pm 0.04^{c}$ \\
\hline $18: 3 n-6$ & $0.39 \pm 0.01^{\mathrm{cd}}$ & $0.64 \pm 0.03^{b}$ & $0.34 \pm 0.01^{\mathrm{cd}}$ & - & - & $0.57 \pm 0.04^{\mathrm{b}}$ \\
\hline $20: 2 n-6$ & - & $0.65 \pm 0.07^{\mathrm{de}}$ & - & - & $0.72 \pm 0.03^{\mathrm{e}}$ & $0.43 \pm 0.04^{\mathrm{cd}}$ \\
\hline $20: 3 n-6$ & - & $0.21 \pm 0.03^{\mathrm{a}}$ & - & - & - & - \\
\hline $20: 4 n-6$ & $0.37 \pm 0.02^{\mathrm{a}}$ & $0.33 \pm 0.03^{\mathrm{a}}$ & $0.53 \pm 0.04^{b}$ & - & $0.37 \pm 0.02^{\mathrm{a}}$ & $0.59 \pm 0.01^{\mathrm{c}}$ \\
\hline $20: 5$ n-3 (EPA) & $14.64 \pm 0.33^{\mathrm{bc}}$ & $14.07 \pm 0.15^{b}$ & $11.31 \pm 0.10^{\mathrm{d}}$ & $14.22 \pm 0.74^{b}$ & $14.64 \pm 1.06^{\mathrm{bc}}$ & $16.48 \pm 0.24^{\text {ca }}$ \\
\hline $22: 5 n-3$ & $0.59 \pm 0.04^{\mathrm{a}}$ & $0.63 \pm 0.09^{\mathrm{a}}$ & $0.56 \pm 0.07^{\mathrm{a}}$ & - & $0.64 \pm 0.05^{\mathrm{a}}$ & $0.72 \pm 0.03^{\mathrm{a}}$ \\
\hline 22:6 n-3 (DHA) & $16.75 \pm 0.36^{\mathrm{a}}$ & $18.21 \pm 0.69^{\mathrm{a}}$ & $18.08 \pm 0.34^{\mathrm{a}}$ & $31.91 \pm 1.43^{\mathrm{e}}$ & $30,57 \pm 1.12^{\mathrm{e}}$ & $25.35 \pm 1.11^{\mathrm{bd}}$ \\
\hline$\sum$ PUFA & $37.00 \pm 0.73^{\text {bd }}$ & $38.64 \pm 0.61^{b}$ & $33.91 \pm 0.48^{\mathrm{d}}$ & $48.81 \pm 1.32^{\mathrm{a}}$ & $49.77 \pm 2.03^{\mathrm{a}}$ & $47.40 \pm 1.15^{\mathrm{ac}}$ \\
\hline$\sum$ HUFA & $32.35 \pm 0.72^{\mathrm{bc}}$ & $34.11 \pm 0.63^{b}$ & $30.47 \pm 0.42^{\mathrm{c}}$ & $46.13 \pm 1.25^{\mathrm{a}}$ & $46.94 \pm 2.11^{\mathrm{a}}$ & $43.57 \pm 1.40^{\mathrm{a}}$ \\
\hline DHA/EPA & $1.14 \pm 0.01^{\mathrm{cd}}$ & $1.29 \pm 0.04^{\text {cde }}$ & $1.60 \pm 0.02^{\mathrm{ab}}$ & $2.24 \pm 0.10^{\mathrm{f}}$ & $2.09 \pm 0.13^{\mathrm{f}}$ & $1.54 \pm 0.05^{\mathrm{abf}}$ \\
\hline
\end{tabular}

Means followed by different letters and letter groups in the same row are significantly different $(\mathrm{p}<0.05, \mathrm{n}=3)$, values are means $\pm \mathrm{SD}$ MUFA - Monounsaturated fatty acids; PUFA - Polyunsaturated fatty acids; HUFA - Highly unsaturated fatty acids; SFA - Saturated fatty acids

up to the OMZ. Temperature changes within the range of $6.5-22^{\circ} \mathrm{C}$ during warm seasons within the layer (Besiktepe et al., 1998; Besiktepe et al., 2005) but they live at 5 to $8.5^{\circ} \mathrm{C}$ in winter and spring (Sazhina, 1987). Svetlichny et al. (2000) showed that total metabolism of C. euxinus occupying a deep daytime habitat decreased nearly 8 fold. The ability to decrease energy expenditure is of great importance especially during summer season when chl-a concentration reduces and averages at $0.22 \mathrm{mg} \mathrm{m}^{-3}$ (Yunev et al., 2002). Almost same results were obtained during the present study and average chl-a concentration reduced in summer season (June, July, August) $\left(0.78 \mathrm{mg} \mathrm{m}^{-3}\right)$ and autumn (September, October, November) $\left(0.76 \mathrm{mg} \mathrm{m}^{-3}\right)$ during the sampling period. It increased in spring (March, April, May) $\left(1.20 \mathrm{mg} \mathrm{m}^{-3}\right)$ and winter (December, February, January) $\left(1.56 \mathrm{mg} \mathrm{m}^{-3}\right)$. In this study, C. euxinus reached the highest average total abundance in winter (December, February, January) (1063 ind $\mathrm{m}^{-3}$ ) when chl-a was the highest. In spring (March, April, May), the abundance of $C$. euxinus was the least ( 256 ind $\mathrm{m}^{-3}$ ) when chl-a was second highest during the sampling period. Results of statistical analyses showed that the correlation between total abundance of $C$. euxinus and chl-a, temperature and salinity was not significant $\left(r^{2}=-0.11, r^{2}=0.44\right.$ and $r^{2}=-0.04$ respectively, $\left.p<0.05\right)$. In addition, total female and male $C$. euxinus abundance peaked in February $\left(847,587\right.$ and 169 ind. $\mathrm{m}^{-3}$ respectively) during the sampling period. However, copepodites reached the most abundance in November (107 ind. $\mathrm{m}^{-3}$ ). Ustun (2005) indicated that C. euxinus peaked in July 2002 (1.800 ind. $\mathrm{m}^{-2}$ ) and in February 2003 (5.200 ind. $\mathrm{m}^{-2}$ ) at coastal station in Sinop Bay of the Black Sea. Yildiz and Feyzioglu (2014) reported that Calanus euxinus reached the highest abundance in December 1999 (18.750 ind. $\left.\mathrm{m}^{-2}\right)$, May 2000 (30.890 ind. $\mathrm{m}^{-2}$ ), April 2001 (52.690 ind. $\mathrm{m}^{-2}$ ), February 2002 (20.714 ind. $\mathrm{m}^{-2}$ ), May 2005 and 2006 (15.869 ind. $\mathrm{m}^{-2}$ and 10.868 ind. $\mathrm{m}^{-2}$ ) at coastal station (Trabzon) in the South-eastern Black Sea. Our results are consistent with that of Ustun (2005) and Yildiz and Feyzioglu (2014). However, it is probable that there are several differences in relation to change of environmental conditions, different sampling periods and stations.

During all seasons, $C$. euxinus is able to accumulate large amounts of lipids (especially wax esters) in the body (Yuneva et al., 1997, 1999). In the present study, during the sampling period, C. euxinus contained the highest amount of total lipids in February (7.03\%) and 
the lowest in September (3.02\%). However, seasonally, the average amount of total lipids was higher in summer (June, July, August) (5.82\%) than the other seasons during the present study. Seasonally, the lowest average reading was observed in autumn (September, October, November) (3.99\%). Svetlichny et al. (2006) showed that $C$. euxinus in deep layers of the Black Sea was dominated by pre-diapause and diapausing postmoult copepodite stage $\mathrm{V}$ with small sexually undifferentiated gonads and mean lipid content of $14.1 \%$ of body volume in summer. Additionally, total lipid in terms of $\mathrm{mg}$ ind $^{-1}$ was the highest in February $\left(0.11 \mathrm{mg}^{\text {ind }} \mathrm{d}^{-1}\right)$. Seasonally, the total lipid was highest in winter (December, February, January) $\left(0.086 \mathrm{mg} \mathrm{ind}^{-1}\right)$ and summer $\left(0.083 \mathrm{mg} \mathrm{ind}^{-1}\right)$ at almost the same level. The results presented in Katnerr et al. (1994) are nearly consistent with the present study. It was indicated that the lipid content of Atlantic copepods was higher in summer than the other seasons. Moreno et al. (1979) reported that the lipid content of Paracalanus parvus along the Mar Del Plata coastline, south of Buenos Aires was $2 \%$ in July (corresponding to the winter season in the northern hemisphere) and increased in September (corresponding to the spring season in the northern hemisphere) to $6.8 \%$. The average amount of total lipids of $C$. euxinus was $14.1 \pm 6.0 \%$ at a different station at the South-eastern Black Sea and this proportion decreased to $7.7 \pm 5.1 \%$ in female C. euxinus living in deeper waters, as reported by Svetlich et al. (2006).

Yuneva et al. (1999) reported on total lipids of female C. euxinus at the Southern Black Sea (September 1996) and indicated that the average amount of total lipids of C. euxinus in cyclonic regions $\left(101.9 \mu\right.$ gind $\left.^{-1}\right)$ was greater than that from anticyclonic regions $\left(58.8 \mu \mathrm{g} \mathrm{ind}^{-1}\right)$. It was pointed out that lipid content (wax esters, triacylglycerols) of female $C$. euxinus was correlated with chl-a $\left(\mathrm{r}^{2}=0.92\right.$, $\mathrm{p}<0.05)$. In the present study, the correlation between lipid content $\left(4.87 \%\right.$ and $\left.0.07 \mathrm{mg}^{\text {ind }^{-1}}\right)$ and chl-a concentration was not significant $\left(r^{2}=0.16, r^{2}=0.08\right.$, $\mathrm{p}<0.05$ respectively). The correlation between lipid ( $\%$ and $\mathrm{mg}$ ind $\left.^{-1}\right)$ and chl-a was not significant $\left(\mathrm{r}^{2}=0.16\right.$ and $\mathrm{r}^{2}=0.08$ respectively, $\left.\mathrm{p}<0.05\right)$. Additionally, the correlation between lipid $\left(\%\right.$ and $\mathrm{mg}$ ind $\left.^{-1}\right)$ and temperature was not significant $\left(r^{2}=0.04\right.$ and $r^{2}=0.01$ respectively, $\left.p<0.05\right)$. Similarly, the correlation between lipid ( $\%$ and $\left.\mathrm{mg} \mathrm{ind}^{-1}\right)$ and salinity was also not significant $\left(\mathrm{r}^{2}=-0.29\right.$ and $\mathrm{r}^{2}=-0.02$ respectively, $\mathrm{p}<0.05$ ). Yuneva et al. (1999) indicated that the level of lipid accumulation in Calanus depends on the concentration and composition of the phytoplankton consumed. However, in the present study, it was thought that rather than the composition and concentration of the phytoplankton consumed, differences in location and timeframe of the sampling may be a factor in the level of lipid accumulation. Black
Sea phytoplankton abundance, biomass, and species composition continuously varies depending on seasonal and regional changes (Eker-Develi and Kideys, 2003).

Calanoid copepods have a special importance in the marine food web since they are the most intensive group of zooplankton (Morris, 1971; Ackman et al., 1974; Lee et al., 1971a). Therefore, the large (2-10 mm), lipid-rich Calanus species have been studied extensively. In early literature, Paracalanus parvus in particular is reported to contain abundant amounts of fatty acids 16:0, $16: 1,20: 5$ n-3 and 22:6 n-3 as compared to the other marine copepods (Ackman and Hooper, 1970; Morris, 1971; Lee et al., 1971a; Ackman et al., 1974;). In the present study, $\sum$ SFA varied from $23.47 \%$ to $37.71 \%$ (March-November), $\sum$ MUFA varied from $16.39 \%$ to 29.95\% (March-September) and $\sum$ PUFA varied from 33.91 to $60.14 \%$ (November-March) in C. euxinus. It was observed that 16:0, 16:1 n-7, 18:1 n-9, 20:5 n-3 and 22:6 n-3 were the major fatty acids in $C$. euxinus and the fatty acid composition and content changed continuously depending on the season and the month. This change in fatty acid composition due to temperature is thought to be an adaptation to optimise phase behaviour that is crucial for proper membrane functioning (Hazel and Williams, 1990; Hochachka and Somero, 2002).

Hagen et al. (1993) reported that the major fatty acids in summer were 20:1 n-9, 20:5 n-3, 22:6 n-3, 18:4 n-3, 22:1 n-11 and 16:1 n-7 in Calanus acutus, 22.1 n-11, 22:1 n-9, 16:0, 20:5 n-3 and 22:6 n-3 in Calanus propinguus collected in the Antarctic Weddell Sea in late winter-early spring (October-November). Kattner et al. (1994) suggested that 22:1 n-9 and 22:1 n-11 long chains were the most important fatty acids and created up to $50 \%$ $\sum$ FAME in Calanus propinquus. They also indicated that MUFA (18:1 n-9 and 16:1 n-7) reached high proportions but PUFA is the dominant fatty acid in Rhincalanus gigas. Kattner et al. (1981) measured the fatty acid content of Pseudocalanus elongatus and Calanus finmarchicus. They found that in P. elongates SFA was $54.1 \pm 6.4 \%$, MUFA was $31.0 \pm 7.4 \%$ and PUFA $14.8 \pm 4.9 \%$, and in C. finmarchicus SFA was 52.2 $\pm 7.6 \%$, MUFA $19.1 \pm 2.5 \%$ and PUFA $28.7 \pm 10.1 \%$. As shown, major fatty acids vary in different Calanus species, however 20:5 n-3 and 22:6 $\mathrm{n}-3$ are the main fatty acids and they comprise a large proportion of the PUFA of nauplii and copepodite stages of copepods in the North-western Mediterranean (Kattner et al., 1994). Moreover, it should be noted that food supply is the most important determinant of fatty acid profile changes of copepods as reported by Escribano and Perez (2010).

Goncalves et al. (2012) observed fatty acid content of different zooplankton species in the waters of the Western 
Atlantic off the coast of Portugal and reported that the dominant unsaturated fatty acids of Acartia tonsa were 14:0, 16:0 and 18:0. Fatty acid content of A. clausi and $A$. tonsa had the same predominant MUFA and SFA and A. clausi also had 17:0 (14.95\%) in the spring. Goncalves et al. (2012) also recorded the highest DHA value in summer (13.28\%) and showed that EPA was observed in all seasons except for autumn. The group also indicated that zooplankton species had higher FA concentrations in winter and spring than in summer and autumn. In the present study, the fatty acid composition of $C$. euxinus was predominantly $\sum$ SFA (average of $31.52 \%$ ) and $\sum$ PUFA (average of $43.01 \%$ ) during the sampling period and the average $\sum$ MUFA was $23.72 \%$. C. euxinus had $17: 0$ during all sampling periods except in April, September and December, although the amount $(17: 0)$ was very low (average of $0.50 \%$ ).

Fatty acid groups ( $\sum$ SFA, $\sum$ MUFA, $\sum$ PUFA, $\sum$ HUFA) were correlated with temperature. While the amount of $\sum$ SFA and $\sum$ MUFA was directly related to temperature $\left(\mathrm{r}^{2}=0.74\right.$ and $\mathrm{r}^{2}=0.73$ respectively, $\left.\mathrm{p}<0.05\right)$, the amounts of $\sum$ PUFA and $\sum$ HUFA were inversely related to temperature $\left(r^{2}=-0.73\right.$ and $r^{2}=-0.80$ respectively, $\left.p<0.05\right)$. The amount of $\sum$ PUFA and $\sum$ HUFA increased $\left(r^{2}=0.61\right.$ and $\mathrm{r}^{2}=-0.68$ respectively, $\left.\mathrm{p}<0.05\right)$, but the $\sum$ MUFA decreased $\left(\mathrm{r}^{2}=-0.68, \mathrm{p}<0.05\right)$ while chl-a increased. The degree of unsaturation increased as temperature decreased. Jeffries (1970) emphasised that the degree of unsaturation decreases from winter to summer. On the other hand, the correlation between chl-a (as an indicator of phytoplankton biomass) and fatty acid groups was significant with the exception of the $\sum$ SFA. It was determined that there is a negative correlation $\left(\mathrm{r}^{2}=-0.75\right)$ between $\Sigma$ MUFA and chlorophyll-a and the $\Sigma$ PUFA $\left(\mathrm{r}^{2}=0.61\right)$ and DHA/EPA ratio $\left(r^{2}=0.60\right)$ and a positive correlation between DHA $\left(\mathrm{r}^{2}=0.61\right)$ and EPA $\left(\mathrm{r}^{2}=0.63, \mathrm{p}<0.05\right)$. In particular, there is a strong relationship between the EPA and the chl-a concentration and chl-a concentrations are associated with diatoms (Budge et al., 2014). Ozdemir and Ak (2012) have reported that dinoflagellates were the most abundant in April and June and the diatoms in October and March in the South-eastern Black Sea (Trabzon Coast). In this study, it was determined that EPA in C. euxinus (as an indicator of the presence of diatoms) had the highest value in March (18.5\%) and increased in spring. This is thought to be a result of the abundance of diatom species at that time. Diatoms represent $85 \%$ of the total phytoplankton in the Black Sea in October (Eker-Develi et al., 1999). However, EPA and DHA were seasonally at its average lowest level in autumn $(13.34 \% ; 17.68 \%$, respectively). The lowest value of EPA was in November $(11.31 \%)$ and DHA was the lowest in September (16.75\%) during the sampling period. Also, at this time, chl-a had the lowest value $\left(0.76 \mu \mathrm{g} \mathrm{l}^{-1}\right)$. This shows that the phytoplankton, specifically the diatoms and flagellate phytoplankton were the lowest at this time. However, it should be noted that phytoplankton species composition, growth and distribution are controlled by such environmental factors as time-dependent changes of temperature, salinity, density, light intensity and nutrient supply (Uysal, 2002). Therefore, it is likely that there is a difference in abundance of phytoplankton from year to year.

The proportion of DHA was observed to be higher than the proportion of EPA during all sampling period. DHA/EPA varied from 1.07 to 2.24 (July-December). Seasonally, average DHA/EPA was the highest in winter (1.96) and the lowest in spring (1.82). This suggests that the high levels of DHA/EPA during spring and winter in $C$. euxinus, a herbivore, depends on the high phytoplankton concentration since chl-a (which is an indicator of phytoplankton biomass) was highest in winter $\left(1.56 \mu \mathrm{g} \mathrm{l}^{-1}\right)$ and spring $\left(1.20 \mu \mathrm{g}^{-1}\right)$. DHA/EPA is a biomarker for carnivorous species (Goncalves et al., 2012). Therefore, the DHA/EPA values of $C$. euxinus can affect fish species that feed on them from larval stages to adult stages in many ways.

Zooplankton forms an essential link between primary producers and higher trophic levels (Dalsgard et al., 2003) and C. euxinus is one of the most important pelagic copepods in the food chain of the Black Sea (Vinogradov et al., 1992). Therefore, the biochemical role of C. euxinus in the Eastern Black Sea is highly significant. C. euxinus has a rich lipid content as well as fatty acid composition and it is a vital link between phytoplankton and commercial fish species (Vinogradov et al., 1992; Kovalev et al., 1998). Results of the study shows that seasonality plays an important role on C. euxinus in the South-eastern Black Sea ecosystem functions, in particular in energy fluxes to higher trophic levels. Lipid content and fatty acid profile of C. euxinus is extremely important in the sustainability of Black Sea anchovy that has an important place in Turkey's fisheries. Because, among the lipids, certain essential fatty acids are considered to be important determinants of ecosystem health and stability (Parrish, 2013). Also, fatty acids provide versatile signatures that are being increasingly employed to delineate the transfer of dietary material through marine and terrestrial food webs (Parrish et al., 2015). C. euxinus is one of the most important food source for anchovy in the Black Sea ecosystem. In this way, it was thought to affect higher trophic levels including anchovy from larval stages to adult stages in many ways such as recruitment, reproduction, growth and survival. Additionally, fatty acid composition of C. euxinus is significantly affected by temperature; the degree of unsaturation is inversely proportional to the temperature. 
If global warming continues, this will have a severe impact on cold water species such as $C$. euxinus and cause losses in the energy transfer in the South-eastern Black Sea food chain, thereby eventually affecting humans.

\section{References}

Ackman, R. G., Linke, B. A. and Hingley, J. 1974. Some details of fatty acids and alcohols in the lipids of North Atlantic copepods. J. Fish. Res. Board Can., 31: 1812-1818.

Ackman, R. G. and Hooper, S. N. 1970. Analysis of fatty acids from Newfoundland copepods and seawater with remarks on the occurrence of arachidic acid. Lipids, 5: 417-421.

Adams, S. M. 1999. Lipids in freshwater ecosystems. In: Arts, M. T. and Wainman, B. C. (Eds.), Ecological role of lipids in the health and success of fish populations, $1^{\text {st }}$ edn. Springer, New York, p. 132-160.

Ahlgren, G., Gustafsson, I. B. and Boberg, M. 1992. Fatty acid content and chemical composition of freshwater microalgae. J Phycol, 28: 37-50.

Arts, M. T., Ackman, R. G. and Holub, B. J. 2001. "Essential fatty acids" in aquatic ecosystems: a crucial link between diet and human health and evolution. Can J. Fish Aquat Sci., 58: 122-137.

Arts, M. T., Brett, M. T. and Kainz, M. J. 2009. Lipids in aquatic ecosystems, Springer, Dordrecht, The Netherlands, 377 pp.

Avsar, D. 1993. The biology and population dynamical parameters of the sprat (Sprattus sprattus phalericus Risso) on the southern coast of the Black Sea. Ph.D. Thesis Cukurova University, Adana, Turkey.

Bat, L. and Satilmis, H. H. 2010. Nutritional condition and migration of the Black Sea anchovy. Yunus Research Bulletin, 2: 11-13.

Besiktepe, S., Kideys, A. E. and Unsal, M. 1998. In situ grasing pressure and diel vertical migration of female Calanus euxinus in the Black Sea. Hydrobiologia, 363: 323-332.

Besiktepe, S., Svetlichny, L., Yuneva, T., Romanova, Z. and Shulman, G. 2005. Diurnal gut pigment rhythm and metabolic rate of Calanus euxinus in the Black Sea. Mar Biol., 146: 1189-1198.

Bonnet, D., Richardson, A. J., Harris, R., Hirst, A., Beaugrand, G., Edwards, M. and Ceballos, S. 2005. An overview of Calanus helgolandicus ecology in European waters. Prog Oceanogr, 65: 1-53.

Budge, S. M., Devred, E., Forget, M., Stuart, V., Trzcinski, M. K., Sathyendranath, S. and Platt, T. 2014. Estimating concentrations of essential omega-3 fatty acids in the ocean: supply and demand. ICES JMar Sci., : 1-9.

Ceballos, S., Cabal, J. A. and Alvarez-Marques, F. 2004. Reproductive strategy of Calanoides carinatus and Calanus helgolandicus during a summer upwelling event off NW Spain. Mar Biol., 145: 739-750.
Christie, W. W. 1990. Gas chromatography and lipids. The Oil Press, Glagow.

Dalsgaard, J., St John M., Kattner, G., Muller-Navarra, D. C. and Hagen, W. 2003. Fatty acid trophic markers in the pelagic marine food environment. Adv. Mar. Biol., 46: 226-340.

Eker-Develi, E. and Kideys, A. E. 1999. Phytoplankton distribution in the western and eastern Black Sea in spring and autumn 1995. ICES J Mar Sci., 56: 15-22.

Eker-Develi, E. and Kideys, A. E. 2003. Distribution of phytoplankton in the southern Black Sea in summer 1996, spring and autumn 1998. J Marine Syst, 39(3-4): 203-221.

Estevez, A., McEvoy, L. A., Bell, J. G. and Sargent, J. R. 1999. Growth, survival, lipid composition and pigmentation of turbot (Scophthalmus maximus) larvae fed live-prey enriched in arachidonic and eicosapentaenoic acids. Aquaculture, 180: 321-343.

Escribano, R. and Perez, C. S. 2010. Variability in fatty acids of two marine copepods upon changing food supply in the coastal upwelling zone off Chile: importance of the picoplankton and nanoplankton fractions. J. Mar. Biol. Ass. U. K. 90(2): 301-313.

Folch, J., Lees, M. and Sloane-Stanley, G. H. 1957. A simple method for the isolation and purification of total lipids from animal tissues. J. Biol. Chem., 226: 497-509.

Goncalves, A. M. M., Azeiteiro, U. M., Pardal, M. A. and De Troch, M. 2012. Fatty acid profiling reveals seasonal and spatial shifts in zooplankton diet in a temperate estuary. Estuar. Coast. Shelf Sci., 109: 70-80.

Hagen, W., Kattner, G. and Graeve, M. 1993. Calanoides acutus and Calanus propinquus, Antarctic copepods with different lipid storage modes via wax esters or triacylglycerols. Mar. Ecol. Prog. Ser., 97: 135-142.

Harris, R., Wiebe, P., Lenz, J., Skjoldal, H. R. and Huntley, M. 2000. ICES Zooplankton methodology manual, Academic Press, UK, 684 pp.

Hazel, J. R. and Williams, E. E. 1990. The role of alterations in membrane lipid composition in enabling physiological adaptation of organisms to their physical environment. Prog. Lipid Res., 29: 167-227

Hochachka, P. W., Somero, G. N. 2002. Biochemical adaptation: mechanism and process in physiological evolution. Oxford University Press, New York.

Jeffries, H. P. 1970. Seasonal composition of temperate plankton communities: fatty acids. Limnol Oceanogr., 15(3): 419-426.

Johnson, W. S. and Allen, D. M. 2005. Zooplankton of the Atlantic and Gulf coasts: A Guide to their identification and ecology. Johns Hopkins University Press, Baltimore, MD, USA.

Kates, M. 1986. Techniques of lipidology : isolation, analysis and identification of lipids, $2^{\text {nd }}$ edn. Laboratory techniques 
in Biochemistry and Molecular Biology 3(2), Elsevier, New York, 464 pp.

Kattner, G., Graeve, M. and Hagen, W. 1994. Ontogenetic and seasonal changes in lipid and fatty acid/alcohol compositions of the dominant Antarctic copepods Calanus propinguus, Calanoides acutus and Rhincalanus gigas. Mar. Biol., 118: 637-644.

Kattner, G., Krause, M. and Trahms, J. 1981. Lipid composition of some typical North Sea copepods, Mar. Ecol. Prog. Ser., 4: 69-74.

Kovalev, A. V., Gubanova, A. D., Kideys, A. E., Melnikov, V. V., Niermann, U., Ostrovskaya, N. A., Skryabin, V.A., Uysal, Z. and Zagorodnyaya, Yu A. 1998. Long-term changes in the biomass and composition of fodder zooplankton in coastal regions of the Black Sea during the period 1957-1996. In: Ivanov, L. and Oguz, T. (Eds.), NATO TUBlack Sea Project: Ecosystem Modeling as a Management Tool for the Black Sea, Symposium on Scientific Results, Kluwer Academic Publishers, p. 209-220.

Latif, M. A., Ozsoy, E., Oguz, T. and Unluata, U. 1991. Observations of the Mediterranean inflow into the Black Sea. Deep-Sea Res., 38: S711-S724.

Lee, R. F., Hirota, J. and Barnett, A. M. 1971a. Distribution and importance of wax esters in marine copepods and in other zooplankton. Deep- Sea Res., 18: 1147-1165.

Lee, R. F., Nevenzel, J. C. and Paffenhoger, G. A. 1971 b. Importance of wax esters and other lipids in the marine food chain: phytoplankton and copepods. Mar. Biol., 9: 99-108.

Lee, R. F. and ve Hirota, J. 1973. Wax esters in tropical zooplankton and nekton and geographical distribution of wax ester in marine copepods. Limnol. Oceanogr., 18: $227-239$.

Marshall, S. M. and Orr, A. P. 1972. The biology of a marine copepod. Reprint. Springer Verlag, Berlin, 195 pp.

Mauchline, J., Blaxter, J. H. S., Southward, A. J. and Tyler, P. A. 1998. The biology of calanoid copepods. $A d v$ Mar. Biol., Academic Press, San Diego, 678 pp.

McEvoy, L. A., Estevez, A., Bell, J. G., Shields, R. J., Gara, B. and Sargent, J. R. 1998. Influence of dietary levels of eicosapentaenoic and arachidonic acids on the pigmentation success of turbot (Scophthalmus maximus L.) and halibut (Hippoglossus hippoglossus L.). Bull. Aquac. Ass. Can., 98(4): 17-20.

Moreno, V. J., Moreno J. E. A. and Brenner, R. R. 1979. Lipid composition of Paracalanus parvus, Oceanologica Acta, 2(4): 373-378.

Morris, R. J. 1971. Comparison of the composition of oceanic copepods from different depths. Comp. Biochem. Physiol., 40B: 275-281.
Muller-Navarra, D. C. 1995. Evidence that a highly unsaturated fatty acid limits Daphnia growth in nature. Arch. Hydrobiol., 132: 297-307.

Muller-Navarra, D. C., Brett, M. T., Liston, A. and Goldman, C. R. 2000. A highlyunsaturated fatty acid predicts biomass transfer between primary producers and consumers. Nature, 403: 74-77.

Oguz, T., La Violette, P. E. and Unluata, U. 1992. The Black Sea circulation: its variability as inferred from hydrographic and satellite observations. J. Geophys. Res., 97: 12569-12584.

Oguz, T., Ducklow, H. W., Malanotte-Rizzoli, P., Murray, J. W., Shushkina, E. A., Vedernikov, V. I. and Unluata, U. 1999. A physical-biochemical model of plankton productivity and nitrogen cycling in the Black Sea. Deep Sea Res., 46: $597-636$

Olsen, Y. 1999. Lipids in freshwater ecosystems In: Arts, M. T. and Wainman, B. C. (Eds.), Lipids and essential fatty acids in aquatic food webs: what can freshwater ecologists learn from mariculture? Springer, New York, p. 161-202.

Ovchinnikov, I. M. and Popov Yu, I. 1987. Evolution of the cold intermediate layer in the Black Sea. Oceanol., 27: 555-560.

Ozdemir, G. P. and Ak, O. 2012. The qualitative and quantitative distribution of the zooplankton in the Southestern Black Sea (Trabzon Coast). J. Black Sea/Mediterranean Env., 18(3): 279-298.

Parrish, C. C. 2013. Lipids in marine ecosystems. ISRN Oceanography, 2013: 1-16.

Parrish, C. C., Nichols, P. D., Pethybridge, H. and Young, J. W. 2015. Direct determination of fatty acids in fish tissues: quantifying top predator trophic connections. Oeecologia, 177: 85-95.

Parsons, T. R., Maita, Y. and Lalli, C. M. 1984. A manual of chemical and biological methods for seawater analysis. Pergamon Press, Great Britain, 173 pp.

Ravet, J. L., Brett, M. T. and Muller-Navarra, D. C. 2003. A test of the role of polyunsaturated fatty acids in phytoplankton food quality for Daphnia using liposome supplementation. Limnol. Oceanogr., 48: 1938-1947.

Rey-Rassat, C., Bonnet, D., Irigoien, X., Harris, R., Head, R. and Carlotti, F. 2004. Secondary production of Calanus helgolandicus in the Western English Channel. J. Ex. Mar. Biol. Ecol., 313: 29-46.

Sargent, J. R., McEvoy, L., Estevez, A., Bell, G., Bell, M., Henderson, J. and Tocher, D. 1999. Lipid nutrition of marine fish during early development: current status and future directions. Aquaculture, 179: 217-229.

Sazhina, L. I. 1987. Reproduction growth and productivity of marine copepoda. Naukova Dumka, Kiev, 156 pp. (in Russian).

Shaffer, G. 1986. Phosphate pumps and shuttles in the Black Sea. Nature, 321: 515-517. 
Simopoulos, A. P. 1999. Essential fatty acids in health and chronic disease. Am. J. Clin. Nutr., 70: 560S-569S.

Sirotenko, D. and Sorokalit, L. K. 1979. Seasonal changes in the food of the Mediterranean sprat, Sprattus sprattus phalericus. J. Ichthyol., 19: 37-51.

Sirotenko, M. D. and Danilevskiy, N. N. 1977. Quantitative indices of the feeding of the Black Sea anchovy, Engraulis encrasicholus ponticus. J. Ichthyol., 17: 610-615.

Stoline, M. R. 1981. The status of multiple comparisons: Simultaneous estimation of all pairwise comparisons in one-way ANOVA designs. Am. Stat., 35(3): 134-141.

Svetlichny, L. S., Hubareva, E. S., Erkan, F. and Gucu, A. G. 2000. Physiological and behavioural aspects of Calanus euxinus females (Copepoda, Calanoida) during vertical migration. Mar. Biol., 137: 963-971.

Svetlichny, L. S., Kideys, A. E., Hubareva, E. S., Besiktepe, S. and Isinibilir, M. 2006. Development and storage in Calanus euxinus from the Black and Marmara seas: Variabilities due to habitat conditions. J. Mar. Syst., 59: 52-62.

Svetlichny, L. S., Yuneva, T. V., Hubareva, E. S., Schepkina, A. M., Besiktepe, S., Kideys, A. E., Bat, L. and Sahin, F. 2009. Development of Calanus euxinus during spring cold homothermy in the Black Sea. Mar. Ecol. Prog. Ser., 374: 199-213.

Tolmazin, D. 1985. Changing coastal oceanography of the Black Sea - I. North-western Shelf. Prog. Oceanogr., 15: 217-276.

TUIK, 2008. State fisheries statistics. TUIK, Turkey

Uysal, Z. 2002. On the formation of net phytoplankton patches in the Southern Black Sea during the spring. Hydrobiologia, 485: 173-182.
Ustun, F. 2005. The composition and seasonal distribution of zooplankton in the region of Sinop Cape of the Black Sea. M. Sc. Disssertaion, Ondokuz Mayıs University, Samsun, Turkey (in Turkish).

Vinogradov, M. E., Arashkevich, E. G. and IIchenko, S. V. 1992. The ecology of the Calanus euxinus population in the deeper layer of its concentration in the Black Sea. J. Plankton Res., 14 (3): 447-458.

Volkman, J. K., Jeffrey, S. W., Nichols, P. D., Rogers, G. I. and Garland, C. D. 1989. Fatty-acid and lipid composition of 10 species of microalgae used in mariculture. J. Exp. Mar. Biol. Ecol., 128: 219-240.

.Y1ldı, I. and Feyzioglu, A. M. 2014. Biological diversity and seasonal variation of mesozooplankton in the Southeastern Black Sea coastal ecosystem. Turkish J. Zool., 38: 179-10.

Yunev, O. A., Suetin, V., Suslin, V. and Moncheva, S. 2002. Long-term changes in the Black Sea surface chlorophyll-a according to in situ and modern satellite data. In: Dahlin, H., Fleming, N. C., Nittis, K. and Petersson, S. E. (Eds.), Elsevier Oceanography Series 69. Building the European capacity in operational oceanography, Proceedings of the Third International Conference on EuroGOOS, 3 - 6 December 2002, Athens, Greece, p. 168-173.

Yuneva, T. V., Svetlichny, L. S., Yunev, O. A., Georgieva, L. V. and Senichkina, L. G. 1997. Spatial variability of Calanus euxinus lipid content in connection with chlorophyll concentration and phytoplankton biomass. Oceanol., 37(5): $745-752$.

Yuneva, T. V., Svetlichny, L. S., Yunev, O. A., Romanova, Z. A., Kideys, A. E., Bingel, F., Uysa1, Z., Yilmaz, A. and Shulman, G. E. 1999. Nutritional condition of female Calanus euxinus from cyclonic and anticyclonic regions of the Black Sea. Mar. Ecol. Prog. Ser., 189: 195-204. 